\title{
Extracorporeal Membrane Oxygenation for ARDS: National Trends in the United States 2008-2012
}

\author{
Bhupinder S Natt MD, Hem Desai MD MPH, Nirmal Singh MBBS, Chithra Poongkunran MD, \\ Sairam Parthasarathy MD, and Christian Bime MD MSc
}

\begin{abstract}
INTRODUCTION: Recent advances in technology and protocols have made the use of extracorporeal membrane oxygenation (ECMO) a viable rescue therapy for patients with ARDS who present with refractory hypoxemia. Despite the lack of strong evidence supporting the use of ECMO in ARDS, its use seems to be increasing. We sought to determine recent trends in the use of ECMO for ARDS. We also assessed trends in mortality among patients with ARDS in whom ECMO was used. METHODS: We performed a retrospective analysis using the largest all-payer in-patient healthcare database in the United States, the Healthcare Cost and Utilization project, the National In-patient Sample database from 2008 to 2012. Subjects with ARDS were identified using carefully chosen International Classification of Diseases, Ninth Revision codes. RESULTS: We found that in 2008, about 1 in 1,000 subjects with ARDS underwent ECMO. Over the subsequent 4-y time period, there was a $0.19 \%$ absolute increase and $70 \%$ relative increase in the use of ECMO for ARDS. The mortality rate among subjects with ARDS in whom ECMO was used declined from $78 \%$ in 2008 to 64\% in 2012. We also found a trend toward a reduction in hospital stay among survivors. CONCLUSION: In the United States, between 2008 and 2012, there was an increasing trend toward the use of ECMO in patients with ARDS that coincided with a slight increase in survival among these patients. Key words: Acute lung injury/ARDS; ECMO; Epidemiology. [Respir Care 2016;61(10):1293-1298. (C) 2016 Daedalus Enterprises]
\end{abstract}

\section{Introduction}

ARDS affects up to 200,000 patients in the United States each year and is a major cause for morbidity and mortality. When Ashbaugh and colleagues first described the syndrome in 1967 , the mortality was about $65 \% .^{1,2}$ Subsequent observational studies have shown a continued trend toward decreasing mortality from ARDS with current estimates at about $30-40 \%$ with severe forms of ARDS

Drs Natt, Bime, and Parthasarathy are affiliated with the Department of Pulmonary, Critical Care, Allergy, and Sleep Medicine, University of Arizona Health Sciences, Tucson, AZ. Drs Desai, Singh and Poongkunran and are affiliated with the Department of Internal Medicine, University of Arizona Health Sciences, Tucson, AZ.

Correspondence: Bhupinder Natt MD, University of Arizona Health Sciences, Division of Pulmonary, Allergy, Critical Care and Sleep, 1501 North Campbell Ave, Tucson, AZ 85724. E-mail: bnatt@ deptofmed.arizona.edu.

DOI: $10.4187 /$ respcare. 04760 having higher mortality rates compared with mild or moderate forms of ARDS. ${ }^{3,4}$ Factors associated with the observed decline in ARDS-related mortality include early recognition, advances in treatment of sepsis and other associated critical illnesses, lung-protective ventilatory strategies, use of neuromuscular blockade, and prone positioning. $5,6,7$ A mortality rate of $30-40 \%$ for ARDS is still unacceptably high. Therefore, researchers and clinicians continue to seek additional therapies that could potentially improve outcomes of ARDS. A subset of patients with severe ARDS present with refractory hypoxemia that is not resolved with strategies like lung recruitment maneuvers, prone positioning, neuromuscular blockade or rescue therapies like inhaled nitric oxide or high-frequency oscillatory ventilation. ${ }^{2,8-11}$ In these patients, extracorporeal membrane oxygenation (ECMO) is an attractive option. The conventional ventilatory support versus ECMO for severe adult respiratory failure (CESAR) trial showed increased 6-month disability-free survival in subjects who received ECMO. ${ }^{12,13}$ Interest in the use of ECMO for ARDS goes back to the 1970s, and earlier studies showed no 
survival benefit. ${ }^{14,15,16}$ However, in the past decade, substantial improvements have been made in the technology and protocols for ECMO, and thus interest has renewed in this therapeutic option for patients with severe ARDS and refractory hypoxemia. In 2009, the CESAR trial 15 showed that subjects transferred to a center with an ECMO-based management protocol had a survival benefit compared with those who received conventional management without referral. We used a large national database of in-patients in the United States to examine the recent trends in the use of ECMO for ARDS during the period from 2008 to 2012. We also examined demographics and mortality trends among subjects with ARDS who underwent ECMO during that period.

\section{See the Related Editorial on Page 1425}

\section{Methods}

\section{Patient Population}

Institutional review board exemption was obtained for this study. We performed a retrospective analysis using the largest all-payer in-patient healthcare database in the United States, the Healthcare Cost and Utilization project - National In-patient Sample database (HCUP-NIS). ${ }^{17} \mathrm{Un}$ weighted, it includes data from approximately 8 million hospital stays per year, representing a sample of $20 \%$ of the hospitals in the United States. Weighted (when expanded to estimate nationwide discharges), it estimates data corresponding to about 40 million annual hospitalizations. To identify patients with ARDS, we examined all patients in the database from 2008 to 2012 with the following International Classification of Diseases, Ninth Revision (ICD-9) codes 518.82, 518.51, 518.52, 518.53, 518.81, 518.84, 518.7, 518.4, 861.20, 785.52, 995.92 and ICD-9 Procedure Codes 96.70, 96.71, and 96.72.17,18 Patients who underwent ECMO therapy were identified by the ICD-9 procedure code 39.65. (Table 1).

\section{Subject Characteristics}

Age, sex, race, income quartile for the subjects' zip code, hospital discharge status, and hospital stay for survivors were extracted from the NIS database. The NIS provides 6 categories for race/ethnicity, as follows: "White," "Black," "Hispanic," "Asian/Pacific Islander," "Native American," and "other." The median income quartile for the subjects' zip code was used as a surrogate for socioeconomic status (SES). The HCUP-NIS database defines SES based on the following quartiles of median income for the subjects' zip code: quartile $1, \$ 1-\$ 38,999$; quartile 2 , $\$ 39,000-\$ 47,999$; quartile $3, \$ 48,000-\$ 62,999$; and quartile, $4 \geq \$ 63,000$.

\section{QUICK LOOK}

\section{Current knowledge}

ARDS continues to have high morbidity and mortality. Survival in patients with ARDS may be improving, however, Extracorporeal membrane oxygenation (ECMO) can be utilized in patients with severe forms of ARDS. There is a paucity of data regarding extracorporeal support measures in ARDS.

\section{What this paper contributes to our knowledge}

The rates of diagnosis of ARDS are increasing as reported by national in-patient sampling data. The survival rates of subjects with ARDS improved during the study period of 2008 to 2012 . There was an increase in the utilization of ECMO from 2008 to 2012 with an improvement in the survival of subjects requiring it. The length of stay in subjects requiring ECMO decreased. As the number of subjects receiving ECMO increased, there seemed to be no difference in the sex or ethnic distribution.

\section{Statistical Analyses}

Continuous variables were reported as means and standard deviations and categorical variables as percentages. Using $\chi^{2}$ tests for trend testing, we examined the trends in ARDS diagnosis, mortality from ARDS, the use of ECMO for ARDS, and in-hospital mortality among subjects with ARDS who underwent ECMO. Among subjects with ARDS in whom ECMO was used, we examined the trends in hospital stay. Next, we assessed the differences in use of ECMO for ARDS by sex, age, race, and SES. Univariate analyses were performed using Mann-Whitney U tests and $\chi^{2}$ tests. Analyses were conducted using STATA/IC 13.1 (StataCorp; Texas, USA).

\section{Results}

We identified a total of 914,912 subjects who fit our definition of ARDS during the period from 2008 to 2012. Table 2 represents the characteristics of these subjects. In 2008 , mortality from ARDS was $51 \%$, and over the next $4 \mathrm{y}$ there was an absolute mortality reduction of $6.5 \%$ and a relative reduction of $12.7 \%$ (Figure 1, Panel B).

\section{Trends in Use of ECMO for ARDS}

In 2008, only about 1 in 1,000 subjects with ARDS underwent ECMO. During the period from 2008 to 2012, there was a $0.19 \%$ absolute increase and a $70 \%$ relative increase in the use of ECMO as rescue therapy for ARDS. 
Table 1. ICD-9 and CPT Codes Used for Identifying Subjects

\begin{tabular}{cc}
\hline \hline & \multicolumn{1}{c}{ Description } \\
\hline ICD-9 Code & \\
518.81 & Acute respiratory failure \\
785.52 & Septic shock \\
995.92 & Severe sepsis \\
518.82 & Other pulmonary insufficiency, not elsewhere \\
& classified \\
518.51 & Acute respiratory failure following trauma and \\
& surgery \\
518.52 & Other pulmonary insufficiency, not elsewhere \\
& classified, following trauma and surgery \\
518.53 & Acute and chronic respiratory failure following \\
& trauma and surgery \\
518.7 & Transfusion-related acute lung injury (TRALI) \\
518.4 & Acute lung edema, NOS \\
861.20 & Unspecified injury of lung without mention of \\
& open wound into thorax \\
ICD-9 Procedure & \\
Code & Continuous invasive mechanical ventilation of \\
96.70 & unspecified duration \\
96.71 & Continuous invasive mechanical ventilation for \\
& $<96$ consecutive hours \\
96.72 & Continuous invasive mechanical ventilation for \\
39.65 & Extracorporeal membrane oxygenation (ECMO) \\
\hline
\end{tabular}

Table 2. Characteristics of Subjects With ARDS

\begin{tabular}{lc}
\hline \multicolumn{1}{c}{ Characteristics } & Subjects, $n(\%)$ \\
\hline $\begin{array}{l}\text { Age }(y) \\
\quad<55\end{array}$ & $242,518(27)$ \\
$\geq 55$ & $656,566(73)$ \\
Sex & \\
$\quad$ Male & $487,905(53)$ \\
Female & $427,135(47)$ \\
Ethnicity & \\
White & $532,543(64)$ \\
Black & $140,569(17)$ \\
Hispanic & $86,321(10)$ \\
Others & $62,981(8)$ \\
Socio-economic status (quartiles) & \\
1 & $272,035(33)$ \\
2 & $218,128(26)$ \\
3 & $155,705(19)$ \\
4 & $185,270(22)$ \\
& \\
\hline The HCUP-NIS database defines socioeconomic status based on the following quartiles of \\
median income for the patient's zip code: \\
quartile $1=\$ 1-\$ 38,999$ \\
quartile $2=\$ 39,000-\$ 47,999$ & \\
quartile $3=\$ 48,000-\$ 62,999$ & \\
quartile $4=\$ 63,000$ and more & \\
\end{tabular}

The greatest increase in use of ECMO for ARDS occurred from 2008 to $2009(0.09 \%$ to $0.2 \%)$, a $62 \%$ relative increase (Table 3).
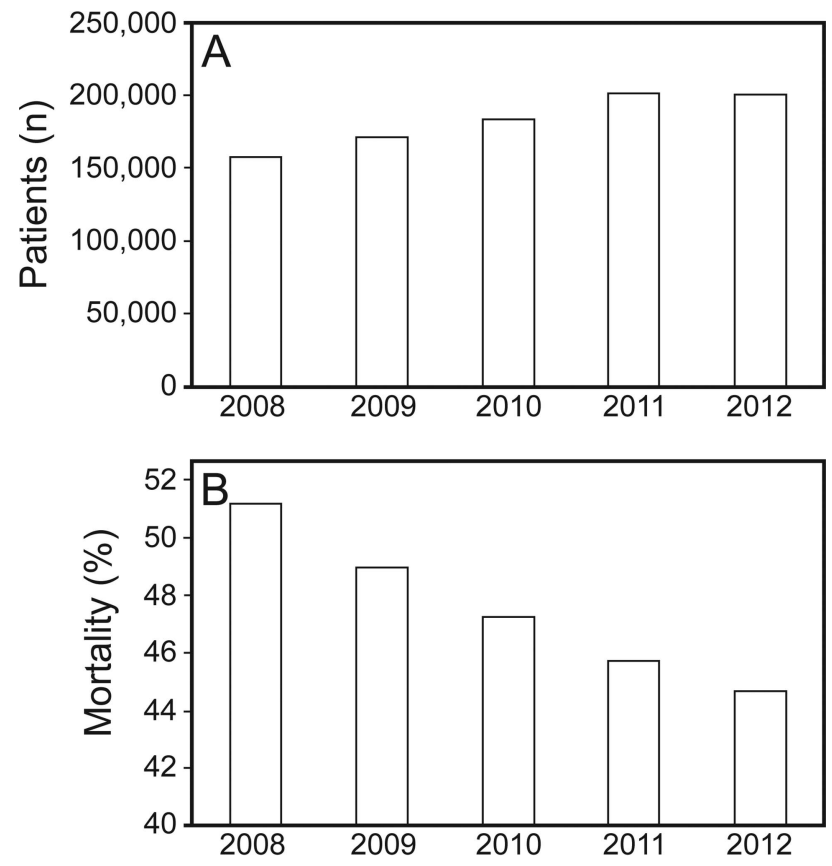

Fig. 1. Overall ARDS prevalence and mortality.

\section{Mortality Trends}

In 2008, the mortality rate among subjects with ARDS in whom ECMO was used was 78\%. During the 5-y period from 2008 to 2012, there was a $14 \%$ absolute reduction and a $19 \%$ relative reduction in mortality. There was a sharp decrease in mortality from 2008 to 2010 (78\% to $64 \%)$. The mortality rate remained stable after 2010 (Table 3).

\section{Hospital Stay}

For subjects with ARDS in whom ECMO was used, the mean hospital stay among survivors decreased from $65 \mathrm{~d}$ in 2009 to $41 \mathrm{~d}$ in 2012. Among nonsurvivors, hospital stay remained relatively stable at between 19 to $23 \mathrm{~d}$.

\section{Use of ECMO for ARDS by Age, Sex, Race, and Socioeconomic Status}

Overall, subjects with ARDS younger than 55 y of age were more likely to be treated using ECMO in the context of ARDS than older subjects were $(1,349 / 242,518 \mathrm{vs}$ $353 / 656,566)(P<.001)$. There were no differences in ECMO utilization by sex (male 973/487,905 vs female $731 / 427,135$ ) or by race (White $827 / 532,543$ vs Black 300/140,569, vs Hispanic 188/86,321). Similarly, there were no differences in the proportion of subjects with ARDS treated with ECMO by socioeconomic status (first quartile $456 / 272,035$ vs second quartile $383 / 218,128$ vs 
Table 3. ECMO Utilization, Mortality and Length of Stay Data

\begin{tabular}{|c|c|c|c|c|c|}
\hline Year & $\begin{array}{c}\text { ARDS } \\
\text { Subjects, } n\end{array}$ & $\begin{array}{c}\text { ECMO } \\
\text { Utilization, } n(\%)\end{array}$ & $\begin{array}{c}\text { ECMO } \\
\text { Mortality, \% }\end{array}$ & $\begin{array}{l}\text { Odds of mortality in } \\
\text { ECMO OR* }(95 \% \mathrm{CI})\end{array}$ & $P$ \\
\hline 2008 & 158,076 & $135(0.085)$ & 78 & 1 & \\
\hline 2009 & 171,473 & $365(0.21)$ & 72 & $0.74(0.45-1.21)$ & .22 \\
\hline 2010 & 183,073 & $270(0.15)$ & 64 & $0.5(0.30-0.80)$ & .004 \\
\hline 2011 & 201,195 & $399(0.20)$ & 57 & $0.38(0.23-0.60)$ & $<.001$ \\
\hline 2012 & 201,095 & $535(0.27)$ & 64 & $0.51(0.32-0.82)$ & .003 \\
\hline \multicolumn{6}{|c|}{$\begin{array}{l}\text { ECMO }=\text { extracorporeal membrane oxygenation. } \\
\text { ECMO Utilization = subject numbers and (percent of subjects). } \\
\text { ECMO mortality = percent mortality among subjects who were treated with ECMO. } \\
* \text { Odds of mortality is in reference to } 2008 \text { year as mortality of ' } 1 \text { ' } \\
\text { Univariate logistic regression was performed comparing mortality in } 2008 \text { (reference) }\end{array}$} \\
\hline
\end{tabular}
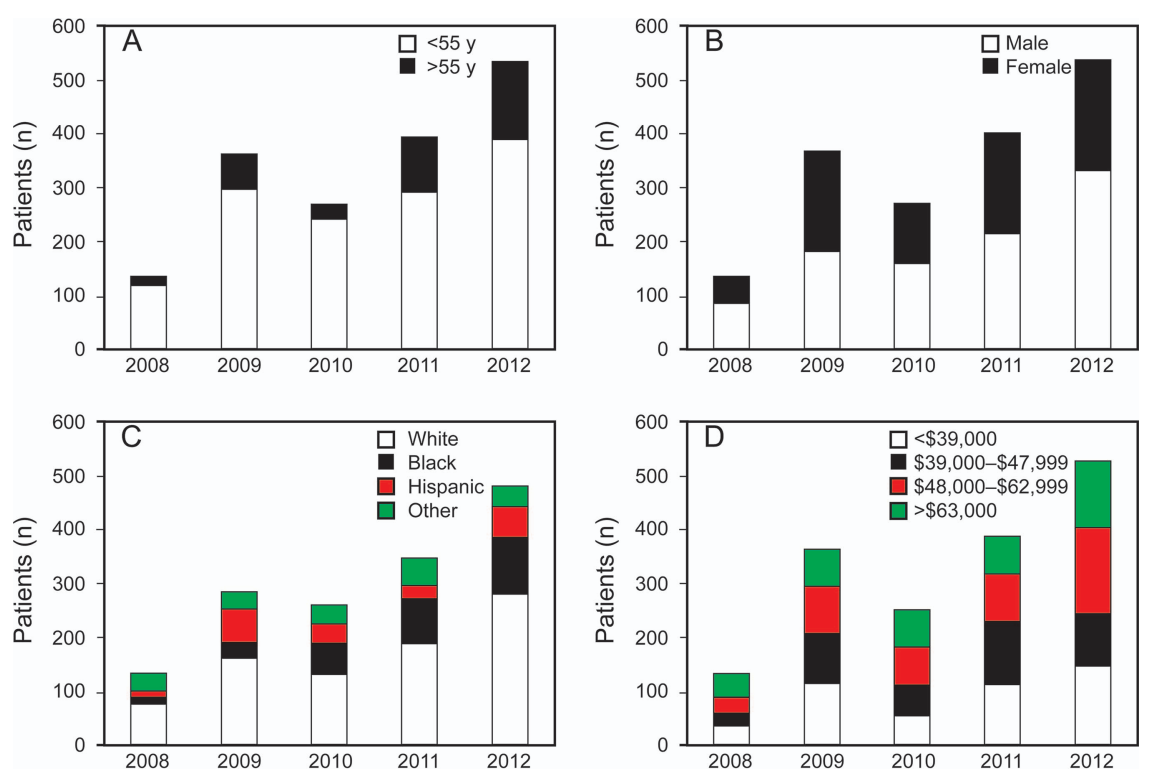

Fig. 2. ECMO Utilization based on age, gender, ethnicity and socioeconomic status.

third quartile 436/155,705 vs fourth quartile 374/185,270). Figure 2 depicts yearly totals of use of ECMO for ARDS by age group, sex, race, and socioeconomic status.

\section{Discussion}

\section{Main Findings}

In this study, we used the largest all-payer in-patient healthcare database in the United States and showed a trend toward increasing use of ECMO for subjects with ARDS from 2008 to 2012. We also found that among patients with ARDS in whom ECMO was used, there was a trend toward decreasing hospital mortality. The hospital stay among survivors decreased during the same period. We did not find any differences in the use of ECMO for ARDS by sex, race, or socioeconomic status.

\section{Strengths and Limitations of This Study}

There are several notable limitations to our analysis. First, we inferred the diagnosis of ARDS based on a combination of ICD-9 codes obtained from a nationwide database that reports diagnoses and procedures for in-patient discharges. Therefore, it is possible that we misclassified some subjects with this method of defining ARDS. However, in such types of large-scale claims data, use of ICD-9 codes for case identification is common. Others have used the HCUP-NIS database and similar claims databases to identify patients with ARDS. ${ }^{18,19}$ The use of ICD-9 procedure codes to identify patients who underwent ECMO is less problematic. However, we could still misclassify subjects who underwent ECMO for reasons other than ARDS. An alternative approach would be to use a prospective database, such as the Extracorporeal Life Support Orga- 
nization (ELSO) database, to identify patients with ARDS who underwent ECMO. ${ }^{20,21}$ However, the ELSO database contains data from 160 United States and 120 international centers that voluntarily supply information and therefore may not be a true representation of yearly national trends in ECMO use for ARDS. A nationwide United Statesbased database like HCUP-NIS, albeit imperfect, gives a good approximation of national trends. Another drawback of using such a database is that severity of illness and the clinical circumstances of patients placed on ECMO cannot be ascertained. A point of novelty for our analysis is the exploration for possible differences in use of ECMO for ARDS by age, race, sex, or socioeconomic status.

\section{Interpretation of Findings in Relation to Previously Published Work}

The yearly total numbers of ECMO use in ARDS from our analysis were similar to those observed in the ELSO database, as reported by Schmidt and colleagues ${ }^{21,22}$ indicating that our algorithm for identifying cases of ECMO in ARDS from the HCUP-NIS database probably performed well. In the ELSO database, the number of ECMO procedures for acute respiratory failure per year was stable at about 100 per year from 2000 to $2008 .^{22}$ In 2009, there was a marked increase to about 350 per year. We observed the same uptick between 2008 and 2009. A likely reason for this sharp increase in use of ECMO for ARDS from 2008 to 2009 was the $2009 \mathrm{H} 1 \mathrm{~N} 1$ influenza pandemic. Numerous reports are available that describe experiences of ECMO use for H1N1-associated ARDS.23,24 There are many possible reasons for the continuous increase in use of ECMO for ARDS since 2009. First, many intensive care units developed expertise in the use of ECMO for ARDS following the experience from the 2009 H1N1 influenza pandemic. Second, improvements have been made in technology and in protocols for the use of ECMO in patients with ARDS. Additionally, strategies for patient selection have improved in recent years ${ }^{23-26}$ Even though the use of ECMO for management of ARDS increased from 2008 to 2012; it is used in fewer than 3 per 1,000 cases of ARDS. If recent trends are an indication, then the use of ECMO for ARDS is expected to continue to rise in the coming years, despite the limited data regarding its efficacy and effectiveness. ${ }^{26,27}$ It is notable that there is a large multicenter phase- 3 trial underway to study the efficacy (60-d mortality) of ECMO in early ARDS versus conventional therapy. ${ }^{28}$

Our findings are also in agreement with those of Schmidt et $\mathrm{al}^{22}$ who showed that the trend in mortality among subjects with ARDS who underwent ECMO decreased slightly between 2008 and 2012. According to their analysis of the ELSO database, the survival rate among subjects with ARDS who underwent ECMO therapy was about $50 \%$ in
2008 and improved to about $60 \%$ in 2009 , followed by a plateau. It is unclear whether this decrease in mortality is due to ECMO therapy or is part of the overall trend of declining ARDS mortality, or both. Alternatively, such a reduction in mortality may be due to administration of ECMO to patients with less severe ARDS who would have a low expected mortality. Ideally, a severity-adjusted analysis may need to be performed to determine the relative contribution of each factor to the observed trend in decreasing mortality.

\section{Implications for Future Research, Policy, and Practice}

The increasing use of ECMO for ARDS brings attention to the need for more evidence of efficacy and effectiveness of this treatment modality that is quite expensive and resource intensive. Ongoing prospective randomized control trials will provide evidence of efficacy, and detailed economic analyses of prospective databases like the ELSO database will give insight into the effectiveness of ECMO for ARDS. These findings also bring attention to the subject of training of current and future critical care physicians in the use of this modality. As more surgical and nonsurgical specialists adopt ECMO for ARDS, rigorous training of physicians along with nursing staff, perfusionists, pharmacists, and other support staff is paramount. With limited resources and staff, innovative multidisciplinary training will need to be developed. Some institutions now offer fellowship training in extracorporeal life support and others have developed local educational programs to train medical care teams.

\section{Conclusions}

We report that in 2009, a sharp increase occurred in the use of ECMO for ARDS and since then, there has been a steady increase. This coincided with a slight increase in survival among subjects with ARDS in whom ECMO was used during the same period. Despite the absence of compelling evidence for its efficacy and effectiveness in ARDS, ECMO is emerging as an important tool for management of severe ARDS with refractory hypoxemia.

\section{REFERENCES}

1. Ashbaugh DG, Bigelow DB, Petty TL, Levine BE. Acute respiratory distress in adults. Lancet 1967;2(7511):319-323.

2. Erickson SE, Martin GS, Davis JL, Matthay MA, Eisner MD for the NIH NHLBI ARDS Network. Recent trends in acute lung injury mortality: 1996-2005. Crit Care Med 2009;37(5):1574-1579.

3. Phua J, Badia JR, Adhikari NKJ, Friedrich JO, Fowler RA, Singh JM et al. Has mortality from acute respiratory distress syndrome decreased over time? AJRCC 2009;179(3):220-27.

4. Bellani G, Laffey JG, Pham T, Fan E, Brochard L, Esteban A et al. Epidemiology, patterns of care, and mortality for patients with acute 


\section{ECMO FOR ARDS: NATIONAL TRENDS}

respiratory distress syndrome in intensive care units in 50 countries. JAMA 2016;315(8):788-800.

5. The Acute Respiratory Distress Syndrome Network. Ventilation with lower tidal volumes as compared with traditional tidal volumes for acute lung injury and the acute respiratory distress syndrome. N Engl J Med 2000;342(18):1301-1308.

6. Papazian L, Forel JM, Gacouin A, Penot-Ragon C, Perrin G, Loundou A et al, ACURASYS Study Investigators. Neuromuscular blockers in early acute respiratory distress syndrome. N Engl J Med 2010; 363(12):1107-1116

7. Guérin C, Reignier J, Richard JC, Beuret P, Gacouin A, Boulain T et al, PROSEVA Study Group. Prone positioning in severe acute respiratory distress syndrome. N Engl J Med 2013;368(23):2159-2168.

8. Guerin C, Debord S, Leray V, Delannoy B, Bayle F, Bourdin G, Richard JC. Efficacy and safety of recruitment maneuvers in acute respiratory distress syndrome. Ann Intensive Care 2011;1(1):9.

9. Dellinger RP, Zimmerman JL, Taylor RW, Straube RC, Hauser DL, Criner GJ et al. Effects of inhaled nitric oxide in patients with acute respiratory distress syndrome: results of a randomized phase II trial. Inhaled Nitric Oxide in ARDS Study Group. Crit Care Med 1998; 26(1):15-23.

10. Afshari A, Brok J, Møller AM, Wetterslev J. Inhaled nitric oxide for acute respiratory distress syndrome (ARDS) and acute lung injury in children and adults. Cochrane Database Syst Rev. 2010;(7): CD002787.

11. Ferguson ND, Cook DJ, Guyatt GH, Mehta S, Hand L, Austin P et al, Canadian Critical Care Trials Group. High-frequency oscillation in early acute respiratory distress syndrome. N Engl J Med 2013; 368(9):795-805

12. Brodie D, Bacchetta M. Extracorporeal membrane oxygenation for ARDS in adults. N Engl J Med 2011;365(20):1905-1914.

13. Peek GJ, Mugford M, Tiruvoipati R, Wilson A, Allen E, Thalanany $\mathrm{MM}$ et al. for the CESAR trial collaboration. Efficacy and economic assessment of conventional ventilatory support versus extracorporeal membrane oxygenation for severe adult respiratory failure (CESAR): a multicenter randomized controlled trial. Lancet 2009;374(9698): 1351-63.

14. Hill JD, O'Brien TG, Murray JJ, Dontigny L, Bramson ML, Osborn JJ, Gerbode F. Prolonged extracorporeal oxygenation for acute posttraumatic respiratory failure (shock lung syndrome). Use of Bramson membrane lung. N Engl J Med 1972;286(12):629-634.

15. Zapol WM, Snider MT, Hill JD, Fallat RJ, Bartlett RH, Edmunds LH et al. Extracorporeal membrane oxygenation in severe acute respiratory failure. JAMA 1979;242(20):2193-2196.
16. Morris AH, Wallace CJ, Menlove RL, Clemmer TP, Orme JF Jr., Dean NC et al. Randomized clinical trial of pressure-controlled inverse ratio ventilation and extracorporeal $\mathrm{CO} 2$ removal for adult respiratory distress syndrome. Am J Respir Crit Care Med 1994; 149(2 Pt 1):295-305.

17. https://www.hcup-us.ahrq.gov/nisoverview.jsp. Last Updated 11/17/ 15. Accessed 12/10/15

18. TenHoor T, Mannino DM, Moss M. Risk Factors for ARDS in the United States: analysis of the 1993 National Mortality Followback Study. Chest 2001;119(4):1179-1184.

19. Stefan MS, Shieh MS, Pekow PS, Rothberg MB, Steingrub JS, Lagu T, Lindenauer PK. Epidemiology and outcomes of acute respiratory failure in the United States, 2001-2009: a national survey. J Hosp Med 2013;8(2):76-82.

20. www.elso.org. Accessed 12/10/15

21. Schmidt M, Bailey M, Sheldrake J, Hodgson C, Auborn C, Rycus PT et al. Predicting survival after extracorporeal membrane oxygenation for severe respiratory failure. Am J Resp Crit Car 2014;189(11):1374-82.

22. http://www.atsjournals.org/doi/suppl/10.1164/rccm.2013112023OC/suppl_file/schmidt_data_supplement.pdf.

23. Noah MA, Peek GJ, Finney SJ, Griffiths MJ, Harrison DA, Grieve $\mathrm{R}$ et al. Referral to an extracorporeal membrane oxygenation center and mortality among patients with severe 2009 influenza (H1N1). JAMA 2011;306(15):1659-68.

24. Australia and New Zealand Extracorporeal Membrane Oxygenation (ANZ ECMO) Influenza Investigators. Extracorporeal membrane oxygenation for 2009 influenza A (H1N1) acute respiratory syndrome. JAMA 2009;302(17):1888-1895.

25. Sharma NS, Wille KM, Zhi D, Thannickal VJ, Brodie DM, Hoopes $\mathrm{CW}$ et al. Use of ECMO in the management of severe acute respiratory distress syndrome: a survey of academic medical centers in the United States. ASAIO J 2015;61:556-563.

26. Combes A, Brodie D, Bartlett R, Brochard L, Brower R, Conard S et al. Position paper for the organization of extracorporeal membrane oxygenation programs for acute respiratory failure in adult patients. Am J Respir Crit Care Med 2014;190:488-496.

27. Morris AH. Exciting new ECMO technology awaits compelling scientific evidence for widespread use in adults with respiratory failure. Intensive Care Med 2012;38(2):186-188.

28. Extracorporeal membrane oxygenator for severe acute respiratory distress syndrome (EOLIA). https://clinicaltrials.gov/ct2/show/ NCT01470703. Accessed 12/10/15. Last updated 11/11/15.

This article is approved for Continuing Respiratory Care Education credit. For information and to obtain your CRCE

(free to AARC members) visit www.rcjournal.com 